\title{
Quality of life of spinal cord injured patients in Taiwan: a subgroup study
}

\author{
Kwan-Hwa Lin ${ }^{1}$, Chy-Ching Chuang ${ }^{1}$, Mu-Jung Kao ${ }^{2}$, I-Nan Lien ${ }^{2}$ and Jau-Yih Tsauo ${ }^{1}$ \\ ${ }^{1}$ School of Physical Therapy, National Taiwan University; ${ }^{2}$ Department of Physical Medicine and Rehabilitation, \\ National Taiwan University Hospital, Taipei, Taiwan, ROC.
}

The major purposes of this study were to assess the quality of life (QOL) of spinal cord injured patients, and to assess the possible factors affecting the QOL. The survey was conducted from 1992-1993 by mailed questionnaires to members of Spinal Cord Injury Association of the Republic of China. There were 347 quality responses with the mean age of $37.5 \pm 10.2$ years old and the mean duration of illness of $7.8 \pm 6.8$ years. The questionnaire included five domains, physical mobility, environment-transportation, psychosocial adjustment, education, and economics for a total of 39 items. Each item contained a rating of 'importance' and 'satisfaction'. The quality of life index (QLI) was calculated by multiplying the satisfaction score with the importance score, then dividing by the possible highest score. The major results included: (1) the subjects had mild to moderate dissatisfaction with most items in five domains except psychosocial adjustment; (2) quality of life in those with complete tetraplegia $(\mathrm{QLI}=-0.41)$ and incomplete tetraplegia $(\mathrm{QLI}=-0.31)$ was significantly lower than that of those with complete paraplegia $(\mathrm{QLI}=-0.13)$ and incomplete paraplegia $(\mathrm{QLI}=-0.04)$; (3) both the severity of injury, and the post-injury working status were the major factors affecting the life quality of spinal cord injured patients in Taiwan.

Keywords: quality of life; spinal cord injury; Taiwan

\section{Introduction}

The life expectancy of persons with spinal cord injury (SCI) has dramatically increased in the past 30 years due to the advancement of medical management. ${ }^{1}$ As a result, many patients with a SCI are surviving to old age and live with a disability which has possibly some impact on their quality of life (QOL). ${ }^{2}$ However, several studies have reported that the perceived quality of life of young and old SCI patients is relatively good. ${ }^{3-6}$

Previous studies have also reported information about factors affecting the quality of life of SCI patients. Siösteen et al investigated 56 SCI's who were about 2.8 years post injury and found that their selfperceived life quality was not related to severity of disability. Cushman and Hassett ${ }^{3}$ evaluated 43 SCI's who were 10 or 15 years post injury and said that neither the level nor the completeness of the injury significantly affected the perceived quality of life. However, they quantify the quality of life with perceived (subjective) quality of life with only one item rated by visual analog scale.

The quality of life is actually conceptualized in multidimensional terms and it has been hypothesized

Correspondence: Kwan-Hwa Lin, RPT, PhD, Associate Professor, School of Physical Therapy, Medical College, National Taiwan University, No. 7, Chung Shan S. Rd., Taipei, Taiwan, R.O.C. to include four large evaluation sectors: behavioral competence, perceived quality of life, objective environment, and psychological well-being ${ }^{7}$. Recently, more researchers ${ }^{8-11}$ have taken a broad perspective of life quality in terms of 'importance' and 'satisfaction' to include the following domains: physical functioning, emotional functioning, sexual functioning, education, social activitites, recreation and economic status.

The exact number of SCI individuals in Taiwan is not known, because there is no national registry of them at this time. Previous epidemiological studies in Taiwan have shown that the incidence rate of those with traumatic SCI was 14.6 per million population in Taipei City, Taiwan, $1978-1981 ;^{12}$ and the incidence rate in the Hualien rural-industrial area in Taiwan was 56.1 per million population, $1986-1990 .{ }^{13}$ Now, the total population in Taiwan (Formosa Island) is 21 million and the estimated new SCI individuals is probably about 1000 persons/year. The official Spinal Cord Injury Association of the Republic of China (SCIAROC) was founded in 1990. The membership is increasing rapidly and has obtained approximately 1000 SCI's from different areas in Taiwan. This number is currently estimated to be only about $1 / 20$ of the whole SCI population in Taiwan, and may represent some characteristics of SCI's who are interested to get in touch with others. 
The purposes of this study were to assess the overall quality of life in five domains, including physical mobility, environment-transportation, psychosocial adjustment, education and economics, and to analyze the factors affecting the life quality in those with a chronic spinal cord injury who are members of SCIAROC. Furthermore, it is reasonable to assume that great dissatisfaction with important items has a more negative impact on life than great dissatisfaction with unimportant items. Therefore, this study quantifies the quality of life in terms of importance and satisfaction.

\section{Methods}

Subjects

Subjects with chronic spinal cord injury were randomly selected from the Spinal Cord Injury Association of the Republic of China (SCIAROC), which recruited approximately 1000 members from different areas in Taiwan. The survey was conducted from December 1992 to June 1993 by mailed questionnaires. The criteria of the subjects were: (1) injured at least one year, and (2) at least 20 years old. There were 347 quality responses (e.g., 179 responses from urban areas and 168 responses from rural areas) with a sample size of 528 subjects, and a response rate of $66 \%$. The major reasons for missing correspondences were: (1) changing the address or phone number; (2) being ill and staying in hospital for treatment; (3) not interested in the issue of quality of life; (4) did not know how to answer. The subjects were not followed up in Rehabilitation Clinics regularly, and most of them came to the clinic only for medication. In this study, the SCI subjects were classified as having complete tetraplegia (CT), incomplete tetraplegia (IT), complete paraplegia (CP), and incomplete paraplegia (IP) according to the classification of ASIA. ${ }^{14}$

\section{Questionnaires}

The questionnaires included three parts: (1) basic data, (2) quality of life assessment, and (3) the suggestions. To further understand the characteristics of this subject, the basic data also contained a multiple choice of questions, such as: the most troublesome complication, and the major device for locomotion following spinal cord injury.

The quality of life questionnaires were constructed according to previous assessment tools, and those questionnaires were modified by the authors to reflect the situations of SCI's in Taiwan. The questionnaires of QOL assessment included five domains: (1) physical mobility, (2) environment-transportation, (3) psychosocial adjustment, (4) education and (5) economics. Totally, thirty-nine items were included in the QOL assessment and each item contained 'importance' and 'satisfaction' evaluation.'
The 14 items of physical mobility were listed according to the Modified Barthel Index ${ }^{15}$ which was valid and reliable for functional impairment assessment. The 12 items of psychosocial adjustment were listed according to the Psychosocial Adjustment to Illness Scale (PAIS), ${ }^{16,17}$ and the suggestions of Trieschmann. ${ }^{18}$ The items in environment, education, and economics were listed according to the current social-economic situations in Taiwan. The questionnaires had been tested on 6 subjects prior to this survey to eliminate any improper questions.

\section{Quality of life index (QLI)}

As suggested by Ferrans and Powers ${ }^{9}$ this study quantified the life quality by calculating the quality of life index (QLI) which was obtained from multiplying the raw score of importance by the recoded score of satisfaction, and the product was divided by the possible highest score. The range of QLI would be between ' +1 ' and ' -1 ', and ' +1 ' indicated the highest level of life quality. The formula of calculating QLI is shown as follows.

$$
\mathrm{QLI}=\frac{\begin{array}{c}
\text { (Importance Raw Score } \times \\
\text { Satisfaction Recoded Score })
\end{array}}{5 \times 7}
$$

The reason to recode the satisfaction score was to distinguish the very satisfied items with low importance from those of the very dissatisfied items with high importance.

Subjects responded to each item on a five-point scale ranging either from 'very important' to 'very unimportant' for the importance items, and from 'very satisfied' to 'very dissatisfied' for the satisfaction items. Score ' 5 ' indicated very important or very satisfied; score '4' indicated quite important or quite satisfied; score ' 3 ' indicated just feel fine (no special preference); score ' 2 ' indicated quite unimportant or quite dissatisfied; and score ' 1 ' indicated very unimportant or very dissatisfied. The five to one points were the raw score of importance and satisfaction. It was reasonable to assume that a subject who had great dissatisfaction with important items would get more negative QLI than a subject who had great dissatisfaction with unimportant items. Therefore, the scoring procedures of QLI in each person included: (1) to recode a satisfaction score by centering the score ' 3 ' on zero; then adding or subtracting 3.5 to the center score for each item, so that ' 5 ', '4', ' 3 ', ' 2 ', ' 1 ' was recorded as ' +7 ', ' +3.5 ', ' 0 ', ' -3.5 ', ' -7 ', respectively; (2) to obtain the score of each item by multiplying the raw importance score with the 'recorded satisfaction score', then dividing by 35 (e.g., $5 \times 7$ ); (3) to obtain the mean score of each domain (e.g., physical mobility etc.) in each person; (4) to obtain the overall QLI score of each person by summing the mean value of five domains then 
dividing by five, so that each domain contributed $20 \%$ of the overall QLI (e.g., to eliminate the bias of uneven item numbers in each domain).

Data analysis

All the raw and recoded data were constructed in the DBASE IV. The statistical analysis was performed by SAS and SPSS/PC. The demographic data were calculated by Crosstabs to obtain frequency and percentage. The one-way ANOVA and NewmanKeuls test were performed to determine the significance of difference in QLI among tetraplegic and paraplegic individuals. The split-half test of each questionnaire was examined by SPSS for the internal reliability, and the correlation coefficient was 0.6 $(P<0.05)$. The stepwise multiple regression analysis was used to analyze the possible factors affecting the life quality. The statistical significance was set at $P<0.05$.

\section{Results}

Basic data

The descriptive measurements of the subjects which indicated the condition at the time of survey are shown in Tables 1, 2 and 3. The characteristics of the patients at survey time are summarized in Table 1. In this survey, the ratio of complete $v s$ incomplete injury was 2.8 , and the ratio of male $v s$ female was 5.9. The mean age of the total sample was $37.5 \pm 10.2$ (mean \pm standard deviation) years old (ranges: $20-71$ y.o.), and there was no significant difference in age among those with complete tetraplegia $(38.0 \pm 10.2$ y.o.), incomplete tetraplegia ( $35.3 \pm 9.0$ y.o.), complete paraplegia $(37.2 \pm 10.1$ y.o.) and incomplete paraplegia (39.2 \pm 11.6 y.o.). The mean duration of illness was $7.8 \pm 6.8$ years (mean \pm standard deviation), and there was no significant difference in duration among those with complete tetraplegia $(7.3 \pm 6.1$ years $)$, incomplete tetraplegia $(6.2 \pm 7.0$ years $)$, complete paraplegia

Table 1 The characteristics of subjects $(n=347)$

\begin{tabular}{|c|c|c|c|c|c|}
\hline & $\begin{array}{c}C T \\
\text { No. }(\%)\end{array}$ & $\begin{array}{c}I T \\
\text { No. }(\%)\end{array}$ & $\begin{array}{c}C P \\
\text { No. }(\%)\end{array}$ & $\begin{array}{c}I P \\
\text { No. }(\%)\end{array}$ & $\begin{array}{c}\text { Total } \\
\text { No. }(\%)\end{array}$ \\
\hline Subject & $71(20.5)$ & $41(11.8)$ & $185(53.3)$ & $50(14.4)$ & $347(100)$ \\
\hline \multicolumn{6}{|l|}{ Sex } \\
\hline Male & $62(17.9)$ & $35(10.1)$ & $157(45.2)$ & $42(12.1)$ & $296(85.3)$ \\
\hline Female & $9(2.7)$ & $6(1.7)$ & $28(8.1)$ & $8(2.3)$ & $51(14.7)$ \\
\hline \multicolumn{6}{|l|}{ Age (y.o.) } \\
\hline $20-39$ & $42(12.1)$ & $30(8.6)$ & $115(33.1)$ & $27(7.2)$ & $214(61.7)$ \\
\hline $40-59$ & $26(7.5)$ & $10(2.9)$ & $66(19.0)$ & $21(6.1)$ & $123(35.4)$ \\
\hline $60-79$ & $3(0.9)$ & $1(0.3)$ & $4(1.2)$ & $2(0.6)$ & $10(2.9)$ \\
\hline \multicolumn{6}{|l|}{ Duration of Illness (year) } \\
\hline $1.0-5.0$ & $35(10.1)$ & $26(7.5)$ & $86(24.8)$ & $28(8.1)$ & $175(50.4)$ \\
\hline $5.1-10.0$ & $19(5.5)$ & $10(2.9)$ & $41(11.8)$ & $13(3.7)$ & $83(23.9)$ \\
\hline$>10.0$ & $17(4.9)$ & $5(1.4)$ & $58(16.7)$ & $9(2.6)$ & $89(25.7)$ \\
\hline \multicolumn{6}{|l|}{ Etiology } \\
\hline MVA & $45(13.0)$ & $25(7.2)$ & $72(20.7)$ & $15(4.3)$ & $157(45.2)$ \\
\hline Fall & $7(2.0)$ & $6(1.7)$ & $53(15.3)$ & $9(2.6)$ & $75(21.6)$ \\
\hline Working injury & $4(1.2)$ & $2(0.6)$ & $29(8.4)$ & $10(2.9)$ & $45(13.0)$ \\
\hline Sports injury & $9(2.6)$ & $2(0.6)$ & $2(0.6)$ & $1(0.3)$ & $14(4.0)$ \\
\hline Others & $6(1.7)$ & $6(1.7)$ & $29(8.4)$ & $15(4.3)$ & $56(16.2)$ \\
\hline \multicolumn{6}{|l|}{ Distribution } \\
\hline City & $39(11.2)$ & $23(6.6)$ & $84(24.2)$ & $33(9.5)$ & $179(51.6)$ \\
\hline Rural area & $32(9.2)$ & $18(5.2)$ & $101(29.1)$ & $17(4.9)$ & $168(48.4)$ \\
\hline \multicolumn{6}{|l|}{ Marital Status } \\
\hline Single & $33(9.5)$ & $16(4.6)$ & $89(25.6)$ & $23(6.6)$ & $161(46.4)$ \\
\hline Married & $30(8.6)$ & $21(6.1)$ & $81(23.3)$ & $23(6.6)$ & $155(44.7)$ \\
\hline Divorced/Widow & $8(2.3)$ & $4(1.2)$ & $15(4.3)$ & $4(1.2)$ & $31(8.9)$ \\
\hline \multicolumn{6}{|l|}{ Education Background } \\
\hline$<$ High school & $37(10.7)$ & $20(5.8)$ & $80(23.1)$ & $22(6.3)$ & $159(45.8)$ \\
\hline High school $\sim$ College & $19(5.5)$ & $12(3.4)$ & $59(17.0)$ & $18(5.2)$ & $108(31.1)$ \\
\hline$\geqslant$ College & $15(4.3)$ & $9(2.6)$ & $46(13.2)$ & $10(2.9)$ & $80(23.1)$ \\
\hline \multicolumn{6}{|l|}{ Employment } \\
\hline Full-time & $3(0.9)$ & $3(0.9)$ & $45(13.0)$ & $16(4.6)$ & $67(19.3)$ \\
\hline Part-time & $2(0.6)$ & $3(0.9)$ & $25(7.2)$ & $6(1.7)$ & $36(10.4)$ \\
\hline Unemployment & $66(19.0)$ & $35(10.1)$ & $115(33.1)$ & $28(8.1)$ & $244(70.3)$ \\
\hline
\end{tabular}

CT: complete tetraplegia; IT: incomplete tetraplegia; CP: complete paraplegia; IP: incomplete paraplegia; MVA: motor vehicle accident 
Table 2 The occuring frequency of most bothering complication after spinal cord injury $(n=347)$

\begin{tabular}{|c|c|c|c|c|c|}
\hline & $\begin{array}{c}C T \\
\text { No. }(\%)\end{array}$ & $\begin{array}{c}I T \\
\text { No. }(\%)\end{array}$ & $\begin{array}{c}C P \\
\text { No. }(\%)\end{array}$ & $\begin{array}{c}I P \\
\text { No. }(\%)\end{array}$ & $\begin{array}{c}\text { Total } \\
\text { No. }(\%)\end{array}$ \\
\hline Urinary problems & $23(6.8)$ & $10(2.9)$ & $60(17.6)$ & $18(5.3)$ & $111(32.0)$ \\
\hline Spasticity & $17(5.0)$ & $11(3.2)$ & $35(10.3)$ & $14(4.1)$ & $77(22.2)$ \\
\hline Numbness/Pain & $11(3.2)$ & $13(3.8)$ & $40(11.8)$ & $13(3.8)$ & $77(22.2)$ \\
\hline Pressure sores & $7(2.1)$ & $1(0.3)$ & $27(7.9)$ & $2(0.6)$ & $37(10.7)$ \\
\hline Contractures & $6(1.8)$ & $3(0.9)$ & $9(2.6)$ & $1(0.3)$ & $19(5.5)$ \\
\hline Others & $7(2.0)$ & $3(0.9)$ & $14(4.0)$ & $2(0.5)$ & $26(7.4)$ \\
\hline
\end{tabular}

CT: complete tetraplegia; IT: incomplete tetraplegia; CP: complete paraplegia; IP: incomplete paraplegia

Table 3 Major device for locomotion after spinal cord injury $(n=347)$

\begin{tabular}{lccrrr}
\hline & $C T$ & $I T$ & $C P$ & IP & Total \\
& No. $(\%)$ & No. $(\%)$ & No. $(\%)$ & No. $(\%)$ & No. $(\%)$ \\
\hline Regular wheelchair & $59(17.0)$ & $26(7.5)$ & $148(42.7)$ & $23(6.6)$ & $256(73.8)$ \\
Electric wheelchair & $12(3.5)$ & $3(0.9)$ & $3(0.9)$ & $0(0.0)$ & $18(5.2)$ \\
Walker & $0(0.0)$ & $2(0.6)$ & $3(0.9)$ & $3(0.9)$ & $8(2.3)$ \\
Crutches & $0(0.0)$ & $9(2.6)$ & $31(8.9)$ & $18(5.2)$ & $58(16.7)$ \\
None & $0(0.0)$ & $1(0.3)$ & $0(0.0)$ & $6(1.7)$ & $7(2.0)$ \\
\hline
\end{tabular}

CT: complete tetraplegia; IT: incomplete tetraplegia; CP: complete paraplegia; IP: incomplete paraplegia

$(8.6 \pm 7.4$ years $)$ and incomplete paraplegia $(6.6 \pm 5.2$ years). Totally, $45.2 \%$ of subjects were injured in a motor vehicle accident (MVA) and $16.2 \%$ was due to non-traumatic injuries such as: tumor, spinal cord apoplexy, etc. There were $51.6 \%$ subjects who came from 16 urban areas and $48.4 \%$ subjects who came from rural areas. The married subjects were $44.7 \%$, and the unemployement rate was $70.3 \%$. Furthermore, $76.9 \%$ subjects had an educational background below college level. In Table 2, the most bothering complication was urinary problems, such as: urinary infection, bladder stone, etc. For locomotion, 73.8\% SCI chose a regular wheelchair as the major device (Table 3).

\section{Importance and satisfaction}

By using the 5- to 1-point scale, the raw scores of importance and satisfaction of the subjects (347 questionnaires) are shown in Tables 4-8. The scores of importance were all above 3-point which indicated 'moderate important to very important'. However, the satisfaction scores varied according to the degree of concern and the severity of injury.

In physical mobility, the most important item was 'feeding yourself independently' and the most dissatisfied item was 'up-down stairs with device independently' (see total in Table 4). Generally, the satisfaction of those who were tetraplegic was less regarding physical mobility than that of those who were paraplegic (Table 4). In environment-transportation, the most important item was 'house-equipped with necessities' and the most dissatisfied item was 'bus equipped with necessities' (see total in Table 5). Most of the satisfaction scores in those with tetraplegia was below ' 2 ' which indicated, 'very dissatisfied' (Table 5).

In psychosocial adjustment, the most important item was, 'having respect from your family and others' and the most dissatisfied item was, 'good sexual function' (see total in Table 6). Therefore, most of the satisfaction scores in those who were tetraplegic and paraplegic were above 3, which indicated, 'moderate to very satisfied' (Table 6).

In education, the most important item was, 'having vocational training' and the most dissatisfied item was, 'school providing special education for the disabled', (see total in Table 7). Totally, most of the satisfaction scores was around ' 2.5 ', which indicated 'moderate dissatisfied to feel fine' (Table 7).

In economics, the most important item was, 'having enough funding by government for assistive devices', and the most dissatisfied item, was 'having enough funding by government for environment or house remodification' (see total in Table 8). In summary, most of the satisfaction score in those with tetraplegia and paraplegia was below ' 3 ' which indicated 'moderate to very dissatisfied' (Table 8).

\section{Quality of life index (QLI)}

After recoding the satisfaction score, the QLI was compared among those with complete tetraplegia, incomplete tetraplegia, complete paraplegia, and incomplete paraplegia. As shown in Figure 1, the QLI for complete tetraplegia $(-0.41 \pm 0.29)$, incomplete tetraplegia $(-0.31 \pm 0.29)$ were significantly $(P<0.05)$ less than that of those with complete paraplegia $(-0.13 \pm 0.25)$ and incomplete paraplegia $(-0.04 \pm 0.29)$. The negative value of QLI indicated 
that their quality of life was below the average (feel fine) level. If comparing the QLI of persons in urban areas $(-0.18 \pm 0.30, n=179)$ to those in rural areas $(-0.20 \pm 0.30, n=168)$ by Student t-test, there was no significant difference. If comparing the QLI of persons with traumatic injury $(-0.19 \pm 0.30, n=291)$ to those with a nontraumatic injury $(-0.21 \pm 0.31, n=56)$, there was also no significant difference.

Table 4 The raw score (mean \pm standard deviation) of importance (I) and satisfaction (S) in the aspect of physical mobility

\begin{tabular}{|c|c|c|c|c|c|c|}
\hline & & $C T$ & $I T$ & $C P$ & $I P$ & Total \\
\hline 1. Feeding yourself & (I) & $4.76 \pm 0.57$ & $4.54 \pm 0.71$ & $4.61 \pm 0.81$ & $4.64 \pm 0.80$ & $4.64 \pm 0.75$ \\
\hline independently & (S) & $2.28 \pm 1.38$ & $3.02 \pm 1.33$ & $4.33 \pm 1.02$ & $4.51 \pm 0.82$ & $3.78 \pm 1.42$ \\
\hline \multirow{2}{*}{$\begin{array}{l}\text { 2. Wearing shirt by } \\
\text { yourself }\end{array}$} & (I) & $4.58 \pm 0.65$ & $4.32 \pm 0.99$ & $4.52 \pm 0.93$ & $4.60 \pm 0.90$ & $4.52 \pm 0.88$ \\
\hline & (S) & $1.61 \pm 1.03$ & $2.71 \pm 1.49$ & $4.24 \pm 1.12$ & $4.54 \pm 0.80$ & $3.56 \pm 1.57$ \\
\hline \multirow{2}{*}{$\begin{array}{l}\text { 3. Wearing pants by } \\
\text { yourself }\end{array}$} & (I) & $4.59 \pm 0.67$ & $4.34 \pm 0.96$ & $4.51 \pm 0.92$ & $4.60 \pm 0.96$ & $4.52 \pm 0.88$ \\
\hline & (S) & $1.45 \pm 0.84$ & $2.54 \pm 1.50$ & $3.85 \pm 1.36$ & $4.11 \pm 1.16$ & $3.23 \pm 1.62$ \\
\hline \multirow[t]{2}{*}{ 4. Bathing yourself } & (I) & $4.51 \pm 0.79$ & $4.33 \pm 0.97$ & $4.43 \pm 0.96$ & $4.65 \pm 0.75$ & $4.48 \pm 0.90$ \\
\hline & (S) & $1.41 \pm 0.84$ & $2.43 \pm 1.48$ & $3.61 \pm 1.35$ & $3.83 \pm 1.21$ & $3.04 \pm 1.56$ \\
\hline \multirow[t]{2}{*}{ 5. Normal urinary control } & (I) & $4.72 \pm 0.61$ & $4.49 \pm 0.89$ & $4.54 \pm 0.94$ & $4.52 \pm 0.93$ & $4.57 \pm 0.87$ \\
\hline & (S) & $1.54 \pm 0.84$ & $2.42 \pm 1.09$ & $2.44 \pm 1.29$ & $2.92 \pm 1.35$ & $2.32 \pm 1.27$ \\
\hline \multirow[t]{2}{*}{ 6. Normal bowel control } & (I) & $4.68 \pm 0.65$ & $4.41 \pm 0.87$ & $4.52 \pm 0.95$ & $4.55 \pm 0.82$ & $4.54 \pm 0.87$ \\
\hline & (S) & $1.49 \pm 0.81$ & $2.45 \pm 1.22$ & $2.53 \pm 1.32$ & $3.00 \pm 1.25$ & $2.37 \pm 1.30$ \\
\hline \multirow[t]{2}{*}{ 7. Rolling independently } & (I) & $4.55 \pm 0.89$ & $4.21 \pm 0.81$ & $4.52 \pm 0.79$ & $4.42 \pm 1.09$ & $4.48 \pm 0.87$ \\
\hline & (S) & $1.83 \pm 1.24$ & $2.98 \pm 1.25$ & $3.91 \pm 1.22$ & $4.16 \pm 0.96$ & $3.41 \pm 1.47$ \\
\hline \multirow[t]{2}{*}{ 8. Transfer independently } & (I) & $4.54 \pm 0.83$ & $4.11 \pm 1.13$ & $4.54 \pm 0.77$ & $4.59 \pm 0.93$ & $4.50 \pm 0.86$ \\
\hline & (S) & $1.52 \pm 0.95$ & $2.71 \pm 1.41$ & $3.74 \pm 1.35$ & $4.13 \pm 1.00$ & $3.22 \pm 1.55$ \\
\hline \multirow{2}{*}{$\begin{array}{c}\text { 9. Wheelchair activity } \\
\text { independently }\end{array}$} & (I) & $4.62 \pm 0.74$ & $4.24 \pm 1.14$ & $4.52 \pm 0.82$ & $4.34 \pm 1.06$ & $4.40 \pm 0.88$ \\
\hline & (S) & $1.91 \pm 1.12$ & $2.61 \pm 1.37$ & $3.79 \pm 1.25$ & $4.12 \pm 0.93$ & $3.32 \pm 1.45$ \\
\hline \multirow{2}{*}{$\begin{array}{l}\text { 10. Ambulation with } \\
\text { device (indoors) }\end{array}$} & (I) & $4.46 \pm 1.04$ & $4.54 \pm 0.74$ & $4.23 \pm 1.12$ & $4.71 \pm 0.65$ & $4.38 \pm 1.02$ \\
\hline & (S) & $1.33 \pm 0.81$ & $1.95 \pm 1.21$ & $2.40 \pm 1.45$ & $3.46 \pm 1.22$ & $2.29 \pm 1.43$ \\
\hline \multirow{2}{*}{$\begin{array}{l}\text { 11. Ambulation with } \\
\text { device (outdoors) }\end{array}$} & (I) & $4.41 \pm 1.05$ & $4.36 \pm 0.93$ & $4.17 \pm 1.20$ & $4.59 \pm 0.72$ & $4.30 \pm 1.09$ \\
\hline & (S) & $1.36 \pm 0.77$ & $1.84 \pm 1.13$ & $2.03 \pm 1.33$ & $3.15 \pm 1.20$ & $2.03 \pm 1.30$ \\
\hline \multirow{2}{*}{$\begin{array}{l}\text { 12. Up-down stairs with } \\
\text { device independently }\end{array}$} & (I) & $4.28 \pm 1.14$ & $4.16 \pm 1.12$ & $3.94 \pm 1.41$ & $4.12 \pm 0.82$ & $4.12 \pm 1.27$ \\
\hline & (S) & $1.29 \pm 0.75$ & $1.79 \pm 1.10$ & $1.51 \pm 1.02$ & $2.82 \pm 1.38$ & $1.70 \pm 1.15$ \\
\hline \multirow{2}{*}{$\begin{array}{l}\text { 13. No complications } \\
\text { (such as: spasticity) }\end{array}$} & (I) & $4.54 \pm 0.83$ & $4.34 \pm 0.79$ & $4.32 \pm 1.08$ & $4.52 \pm 0.97$ & $4.40 \pm 0.99$ \\
\hline & (S) & $1.76 \pm 0.92$ & $1.80 \pm 0.88$ & $2.28 \pm 1.06$ & $2.40 \pm 1.36$ & $2.13 \pm 1.09$ \\
\hline \multirow{2}{*}{ 14. Good home care } & (I) & $4.43 \pm 0.87$ & $3.92 \pm 1.18$ & $3.60 \pm 1.34$ & $3.80 \pm 1.32$ & $3.80 \pm 1.32$ \\
\hline & (S) & $2.28 \pm 1.24$ & $2.32 \pm 1.20$ & $2.49 \pm 1.18$ & $2.50 \pm 1.13$ & $2.43 \pm 1.19$ \\
\hline
\end{tabular}

CT: complete tetraplegia; IT: incomplete tetraplegia; CP: complete paraplegia; IP: incomplete paraplegia. Rating the score by 5-point scale: 5 -very important or very satisfied; 4 -moderate important or moderate satisfied; 3 -just feel fine (no preference); 2 -moderate unimportant or moderate dissatisfied; 1 -very unimportant or very dissatisfied

Table 5 The raw score (mean \pm standard deviation) of importance (I) and satisfaction (S) in the aspect of environmenttransportation

\begin{tabular}{lcccccc}
\hline & & $C T$ & $I T$ & $C P$ & IP & Total \\
\hline 1. House equipped with & (I) & $4.72 \pm 0.74$ & $4.58 \pm 0.68$ & $4.54 \pm 0.86$ & $4.67 \pm 0.03$ & $4.60 \pm 0.79$ \\
$\quad$ necessities & (S) & $2.34 \pm 1.36$ & $2.44 \pm 1.21$ & $2.63 \pm 1.30$ & $3.13 \pm 1.29$ & $2.61 \pm 1.32$ \\
2. Buildings designed for & (I) & $4.63 \pm 0.83$ & $4.56 \pm 0.71$ & $4.44 \pm 1.02$ & $4.66 \pm 0.72$ & $4.53 \pm 0.91$ \\
$\quad$ the disabled & (S) & $1.96 \pm 1.11$ & $1.90 \pm 0.93$ & $2.13 \pm 1.15$ & $2.21 \pm 1.19$ & $2.08 \pm 1.12$ \\
3. Road with smooth & (I) & $4.71 \pm 0.73$ & $4.59 \pm 0.77$ & $4.51 \pm 0.89$ & $4.66 \pm 0.63$ & $4.58 \pm 0.82$ \\
$\quad$ ground & (S) & $2.03 \pm 1.04$ & $1.95 \pm 1.04$ & $2.15 \pm 1.18$ & $2.26 \pm 1.03$ & $2.12 \pm 1.11$ \\
4. Bus equipped with & (I) & $4.67 \pm 0.78$ & $4.50 \pm 0.93$ & $4.52 \pm 0.95$ & $4.73 \pm 0.73$ & $4.58 \pm 0.89$ \\
$\quad$ necessities & (S) & $1.81 \pm 1.02$ & $1.75 \pm 0.84$ & $1.82 \pm 1.03$ & $2.00 \pm 1.27$ & $1.84 \pm 1.05$ \\
5. Enough parking lot & (I) & $4.49 \pm 0.93$ & $4.48 \pm 0.96$ & $4.48 \pm 0.91$ & $4.66 \pm 0.87$ & $4.50 \pm 0.91$ \\
$\quad$ for the disabled & (S) & $1.97 \pm 0.98$ & $1.76 \pm 0.80$ & $2.07 \pm 1.09$ & $1.79 \pm 0.83$ & $1.98 \pm 1.01$ \\
6. Having adaptable & (I) & $4.48 \pm 0.94$ & $4.53 \pm 0.92$ & $4.49 \pm 0.96$ & $4.61 \pm 0.91$ & $4.51 \pm 0.94$ \\
$\quad$ devices for outdoors & (S) & $1.80 \pm 1.08$ & $1.74 \pm 1.02$ & $2.54 \pm 1.42$ & $2.88 \pm 1.44$ & $2.35 \pm 1.38$ \\
\hline
\end{tabular}

CT: complete tetraplegia; IT: incomplete tetraplegia; CP: complete paraplegia; IP: incomplete paraplegia. Rating the score by 5-point scale: 5-very important or very satisfied; 4-moderate important or moderate satisfied; 3 -just feel fine (no preference); 2 -moderate unimportant or moderate dissatisfied; 1 -very unimportant or very dissatisfied 
Table 6 The raw score (mean \pm standard deviation) of importance (I) and satisfaction (S) in the aspect of psychosocial adjustment

\begin{tabular}{llccccc}
\hline & & $C T$ & $I T$ & $C P$ & IP & Total \\
\hline 1. You can accept the & (I) & $4.44 \pm 0.91$ & $4.32 \pm 0.99$ & $4.33 \pm 0.90$ & $4.48 \pm 0.88$ & $4.37 \pm 0.91$ \\
fact of injury & (S) & $3.24 \pm 1.34$ & $2.93 \pm 1.10$ & $3.36 \pm 1.12$ & $3.49 \pm 1.10$ & $3.30 \pm 1.17$ \\
2. You won't get angry & (I) & $4.37 \pm 0.88$ & $4.12 \pm 0.93$ & $4.14 \pm 0.96$ & $4.39 \pm 1.01$ & $4.22 \pm 0.95$ \\
$\quad$ easily & (S) & $2.91 \pm 1.17$ & $3.10 \pm 1.07$ & $3.38 \pm 0.92$ & $3.53 \pm 1.02$ & $3.27 \pm 1.02$ \\
3. Usually you are not & (I) & $4.34 \pm 0.94$ & $4.17 \pm 0.86$ & $4.14 \pm 0.89$ & $4.28 \pm 0.99$ & $4.20 \pm 0.91$ \\
$\quad$ in depression & (S) & $3.14 \pm 1.11$ & $3.00 \pm 0.96$ & $3.31 \pm 0.98$ & $3.31 \pm 1.03$ & $3.24 \pm 1.02$ \\
4. You won't mind curious & (I) & $4.31 \pm 1.04$ & $4.15 \pm 0.92$ & $4.12 \pm 1.02$ & $4.12 \pm 1.15$ & $4.17 \pm 1.03$ \\
inquiry & (S) & $3.04 \pm 1.25$ & $3.05 \pm 1.12$ & $3.28 \pm 1.13$ & $3.48 \pm 1.13$ & $3.23 \pm 1.16$ \\
5. You are not alcoholic & (I) & $4.43 \pm 1.04$ & $4.20 \pm 1.07$ & $4.15 \pm 1.16$ & $4.23 \pm 1.29$ & $4.22 \pm 1.14$ \\
$\quad$ or drug abused & (S) & $3.62 \pm 1.46$ & $3.73 \pm 1.13$ & $3.79 \pm 1.17$ & $3.85 \pm 1.09$ & $3.76 \pm 1.22$ \\
6. Having respect from & (I) & $4.69 \pm 0.60$ & $4.57 \pm 0.63$ & $4.39 \pm 0.86$ & $4.40 \pm 0.86$ & $4.47 \pm 0.80$ \\
$\quad$ your family and others & (S) & $3.49 \pm 1.32$ & $3.85 \pm 0.94$ & $3.84 \pm 1.02$ & $3.71 \pm 1.06$ & $3.75 \pm 1.09$ \\
7. Having good care from & (I) & $4.68 \pm 0.68$ & $4.37 \pm 0.86$ & $4.27 \pm 0.93$ & $4.46 \pm 0.77$ & $4.39 \pm 0.87$ \\
$\quad$ your family and others & (S) & $3.45 \pm 1.37$ & $3.63 \pm 1.10$ & $3.73 \pm 1.00$ & $3.58 \pm 1.09$ & $3.64 \pm 1.16$ \\
8. You are enthusiastic in & (I) & $4.11 \pm 1.09$ & $4.20 \pm 0.81$ & $4.10 \pm 1.00$ & $4.35 \pm 0.86$ & $4.15 \pm 0.98$ \\
$\quad$ public services & (S) & $2.82 \pm 1.25$ & $2.90 \pm 1.16$ & $3.16 \pm 1.09$ & $3.29 \pm 1.10$ & $3.08 \pm 1.14$ \\
9. Encouraging and & (I) & $4.54 \pm 0.73$ & $4.47 \pm 0.73$ & $4.30 \pm 0.84$ & $4.52 \pm 0.74$ & $4.40 \pm 0.80$ \\
$\quad$ helping others & (S) & $3.04 \pm 1.29$ & $3.15 \pm 1.15$ & $3.40 \pm 1.01$ & $3.40 \pm 1.01$ & $3.30 \pm 1.10$ \\
10. Having recreational & (I) & $3.94 \pm 1.30$ & $3.88 \pm 1.05$ & $3.83 \pm 1.16$ & $4.04 \pm 1.21$ & $3.89 \pm 1.18$ \\
$\quad$ activities & (S) & $2.32 \pm 1.24$ & $2.68 \pm 1.16$ & $2.88 \pm 1.17$ & $2.78 \pm 1.23$ & $2.73 \pm 1.21$ \\
11. Good relationship & (I) & $4.31 \pm 1.12$ & $4.15 \pm 1.15$ & $4.35 \pm 0.92$ & $4.39 \pm 0.95$ & $4.32 \pm 0.99$ \\
$\quad$ with others & (S) & $3.10 \pm 1.48$ & $3.13 \pm 1.10$ & $3.33 \pm 1.18$ & $3.34 \pm 1.24$ & $3.22 \pm 1.24$ \\
12. Good sexual function & (I) & $3.82 \pm 1.26$ & $3.92 \pm 1.24$ & $3.74 \pm 1.30$ & $3.92 \pm 1.35$ & $3.80 \pm 1.29$ \\
& (S) & $2.30 \pm 1.17$ & $2.58 \pm 1.08$ & $2.34 \pm 1.13$ & $2.32 \pm 1.14$ & $2.36 \pm 1.13$ \\
\hline
\end{tabular}

CT: complete tetraplegia; IT: incomplete tetraplegia; CP: compete paraplegia; IP: incomplete paraplegia. Rating the score by 5-point scale: 5 -very important or very satisfied; 4 -moderate important or moderate satisfied; 3 -just feel fine (no preference); 2 -moderate unimportant or moderate dissatisfied; 1 -very unimportant or very dissatisfied

Table 7 The raw score (mean \pm standard deviation) of importance (I) and satisfaction (S) in the aspect of education

\begin{tabular}{lcccccc}
\hline & & $C T$ & $I T$ & $C P$ & IP & Total \\
\hline 1. Attending workshop for & (I) & $4.55 \pm 0.81$ & $4.45 \pm 0.90$ & $4.44 \pm 0.76$ & $4.56 \pm 0.84$ & $4.48 \pm 0.80$ \\
$\quad$ basic medical knowledge & (S) & $2.29 \pm 1.12$ & $2.20 \pm 1.02$ & $2.49 \pm 1.09$ & $2.53 \pm 1.18$ & $2.42 \pm 1.10$ \\
2. School providing special & (I) & $4.46 \pm 0.88$ & $4.20 \pm 1.02$ & $4.40 \pm 0.85$ & $4.55 \pm 0.87$ & $4.41 \pm 0.88$ \\
$\quad$ education for the disabled & (S) & $1.83 \pm 0.96$ & $2.11 \pm 1.03$ & $2.27 \pm 1.05$ & $2.36 \pm 1.14$ & $2.17 \pm 1.06$ \\
3. Having vocational & (I) & $4.67 \pm 0.68$ & $4.40 \pm 0.96$ & $4.53 \pm 0.76$ & $4.64 \pm 0.72$ & $4.56 \pm 0.76$ \\
$\quad$ training & (S) & $2.06 \pm 1.16$ & $2.13 \pm 1.04$ & $2.57 \pm 1.06$ & $2.37 \pm 1.04$ & $2.39 \pm 1.09$ \\
\hline
\end{tabular}

CT: complete tetraplegia; IT: incomplete tetraplegia; CP: complete paraplegia; IP: incomplete paraplegia. Rating the score by 5-point scale: 5 -very important or very satisfied; 4 -moderate important or moderate satisfied; 3 -just feel fine (no preference); 2 -moderate unimportant or moderate dissatisfied; 1 -very unimportant or very dissatisfied

Factors affecting quality of life

The possible factors affecting the quality of life were listed as sex, age, location (urban/rural areas), duration of illness, severity of injury, marital status, educational background, post-injury working status and urinary complications. The underlying assumptions of regression analysis were checked and no meaningful violations were apparent. The nonparametric data with three or more groups were assigned with a dummy or a reference group. In post-injury working status, the group without work was set as reference and coded as ' 0 '. The group with part-time work or fulltime work was coded as ' 1 ' during stepwise analysis. In severity of injury, the complete tetraplegics was set as the reference and coded as ' 0 '. Those who had incomplete tetraplegia or complete paraplegia, or incomplete paraplegia was coded as ' 1 ' during stepwise analysis. As shown in Table 9, post-injury working status and severity of injury were the major factors affecting the quality of life, and location and sex did not affect the quality of life significantly, and the contributions from other factors were trivial.

\section{Discussion}

The results of this study indicate: (1) spinal cord injured patients had mild to moderate dissatisfaction with most items in four domains (e.g., physical mobility, environment-transportation, education, and economics) except psychosocial adjustment; (2) the 
quality of life of those who were tetraplegic was significantly lower than was that of those who were paraplegic; (3) both the level of injury and post-injury working status were the major factors influencing the quality of life. However, previous reports mentioned that the quality of life of SCI's was relatively good and was not related to the level of injury. ${ }^{3-6}$ This variance in results might be due to the different method of assessment and/or cultural background.

In our method of assessment, the major differences of this study included: (1) to measure the quality of life in five domains (physical mobility, environmenttransportation, psychosocial adjustment, education, and economics) which were supposed to reflect the overall scope of life quality; (2) to quantify the life quality by quality of life index which could carefully identify the difference of importance and satisfaction in each domain. However, previous studies focused on the assessment of perceived well-being, with only one item qualifying as subjective quality of life. ${ }^{3-6}$

If examining the score of psychosocial adjustment of this study, all the subjects showed 'moderate to very' satisfied in most items (Table 6). Therefore, this result seemed not contradictory to previous reports on the issue of the subjective assessment of perceived wellbeing in SCI. ${ }^{3-6,17-18}$ Furthermore, examining the score of physical mobility of this study, all the subjects showed 'moderate to very' dissatisfied in most items

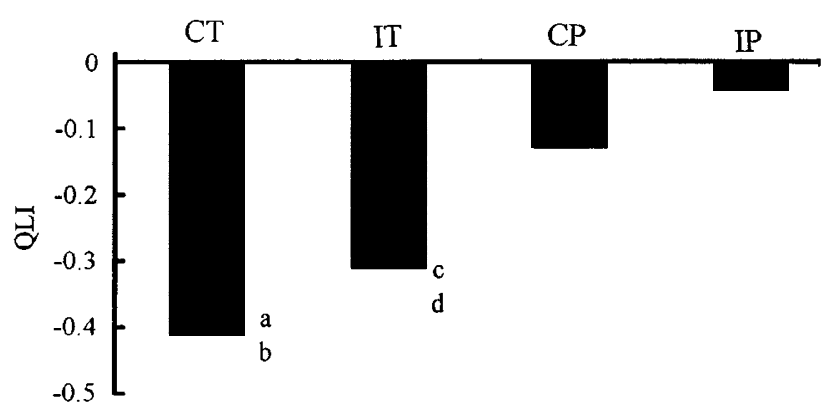

Figure 1 The comparison of quality of life index (QLI) among those who have complete tetraplegia (CT), incomplete tetraplegia (IT), complete paraplegia (CP), and incomplete paraplegia (IP). a: CT $<\mathrm{CP}$ at $\mathrm{p}<0.05$; b:CT $<$ IP at $\mathrm{p}<0.05$; c: IT $<$ CP at $\mathrm{p}<0.05 ; \mathrm{d}$ : IT $<$ IP at $\mathrm{p}<0.05$

Table 8 The raw score (mean \pm standard deviation) of importance (I) and satisfaction (S) in the aspect of economics

\begin{tabular}{lcccccc}
\hline & & $C T$ & $I T$ & $C P$ & IP & Total \\
\hline 1. Having stable job & (I) & $4.55 \pm 0.91$ & $4.32 \pm 1.13$ & $4.53 \pm 0.87$ & $4.57 \pm 1.00$ & $4.51 \pm 0.93$ \\
$\quad$ and income & (S) & $1.71 \pm 1.08$ & $1.93 \pm 1.21$ & $2.22 \pm 1.23$ & $2.65 \pm 1.23$ & $2.14 \pm 1.23$ \\
2. Having enough money for & (I) & $4.51 \pm 0.86$ & $4.41 \pm 1.05$ & $4.48 \pm 0.96$ & $4.56 \pm 0.91$ & $4.49 \pm 0.94$ \\
$\quad$ basic living & (S) & $1.87 \pm 1.08$ & $1.90 \pm 1.15$ & $2.23 \pm 1.19$ & $2.79 \pm 1.29$ & $2.20 \pm 1.21$ \\
3. Having enough funding by & (I) & $4.62 \pm 0.74$ & $4.45 \pm 1.01$ & $4.64 \pm 0.71$ & $4.79 \pm 0.46$ & $4.64 \pm 0.73$ \\
$\quad$ government for assistive & (S) & $2.41 \pm 1.11$ & $2.23 \pm 0.99$ & $2.68 \pm 1.14$ & $2.77 \pm 1.05$ & $2.58 \pm 1.11$ \\
$\quad$ devices & & & & & & \\
4. Having enough funding by & (I) & $4.60 \pm 0.79$ & $4.45 \pm 1.01$ & $4.58 \pm 0.78$ & $4.81 \pm 0.50$ & $4.59 \pm 0.83$ \\
$\quad$ government for enviornment & (S) & $1.93 \pm 1.14$ & $1.73 \pm 0.91$ & $2.04 \pm 1.03$ & $2.22 \pm 1.13$ & $2.01 \pm 1.05$ \\
$\quad$ or house remodification & & & & & &
\end{tabular}

CT: complete tetraplegia; IT: incomplete tetraplegia; CP: complete paraplegia; IP: incomplete paraplegia. Rating the score by 5-point scale: 5 -very important or very satisfied; 4 -moderate important or moderate satisfied; 3 -just feel fine (no preference); 2 -moderate unimportant or moderate dissatisfied; 1 -very unimportant or very dissatisfied

Table 9 Multiple regression analysis for possible factors affecting quality of life

\begin{tabular}{|c|c|c|c|c|c|}
\hline Variable & Beta & $\begin{array}{l}\text { Standard } \\
\text { error }\end{array}$ & $\begin{array}{l}\text { Partial } \\
R^{2}\end{array}$ & $\begin{array}{c}\text { Model } \\
R^{2}\end{array}$ & $P$-value \\
\hline (Intercept) & -2.63 & 0.03 & & & \\
\hline \multicolumn{6}{|l|}{ Post-injury work } \\
\hline Without work & reference & & & & \\
\hline Part-time & 0.72 & 0.20 & 0.013 & 0.013 & $0.02 *$ \\
\hline Full-time & 0.54 & 0.24 & 0.061 & 0.074 & $0.01 *$ \\
\hline \multicolumn{6}{|l|}{ Severity of injury } \\
\hline Complete tetraplegics & references & & & & \\
\hline Incomplete tetraplegics & 0.44 & 0.26 & 0.006 & 0.080 & 0.09 \\
\hline Complete paraplegics & 1.20 & 0.19 & 0.044 & 0.124 & $0.01 *$ \\
\hline Incomplete paraplegics & 1.65 & 0.25 & 0.100 & 0.224 & $0.01 *$ \\
\hline Location & 0.19 & 0.15 & 0.004 & 0.228 & 0.22 \\
\hline Sex & 0.23 & 0.20 & 0.003 & 0.231 & 0.27 \\
\hline
\end{tabular}

*Indicates highly significant. Partial $\mathrm{R}^{2}$ : the correlation of quality of life in each variable. Model $\mathrm{R}^{2}$ : the accumulated value from partial $\mathrm{R}^{2}$ to show the predictive accuracy of quality of life 
and the scores were related to the level of injury (Table 4). This result was similar to the report of Siösteen et al on the objective assessment of Modified Barthel Index (self-care and mobility index) of spinal cord injured patients. ${ }^{6}$ Therefore, it is better to interpret the results with the explanation of our evaluation methods.

In terms of the cultural background, a difference between oriental (Taiwan) and western countries exists. In Taiwan, the economic and social support systems for the disabled person are mainly from the familiy and/or relatives, while the community rehabilitation and nursing home care systems are not well established. The living stipend for the disabled offered by the government is around the minimum living standard in Taiwan. Also, there are some limitations in funding for necessities. Therefore, the satisfaction for family support was around 3.7 (see psychosocial adjustment in Table 6), but the satisfaction for government funding was around 2.1 (see economics in Table 8). Furthermore, it was noticed that the rating for the sexual importance was not as high as the other aspects of life in this study (see psychosocial adjustment in Table 6). The study of White et al ${ }^{11}$ in Texas also showed that 79 SCI's rated the importance of sexual life in the lowest rank as compared to others of life, such as family relationship, and recreational activities. The cultural or religious influence on this issue might require further investigation. However, decrease of sexual drive in spinal cord injured patients has been reported. ${ }^{18}$

The major factors affecting the quality of life are severity of injury and the postinjury work status (Table 9), and those two factors can explain $22.4 \%$ of the life quality. The next possible factors, such as location and sex, can explain only about $0.7 \%$ more of the life quality. For future study, the influence of estate on life quality may have to be taken into consideration. This study indicated that the working opportunity for the disabled is highly limited. However, the relationship between postinjury work status and quality of life may not be the true causeeffect phenomenon, because the reverse is equally possible. Therefore, a comparison of the life quality before and after injury is suggested for future studies.

The satisfaction on environment-transportation was low in this survey (see environment-transportation in Table 5). At the time of survey, most of the new buildings or buses have the set-up for disabled persons, but the older buildings or buses do not incorporate these features. In this survey, the major suggestions from the spinal cord injured persons were: (1) to provide enough funding or loans for persons who want to develop his/her own career; (2) to simplify the procedures and reduce limitations for application of assistive devices or house remodeling; (3) to establish the proper welfare system for the disabled.

The major limitations of this survey are that the response rate was low and the validity of the questionnaire was not well-established. The low response rate may be due to the complexity of the questions compounded by the ignorance of this type of issue by some people. However, the validity is shown indirectly in this survey by having all of the items perceived by our staff to be important to the patients. The other limitation in this study is that this study only solicited the members of the SCI association, and may over estimate the life quality of the spinal cord injured patients in Taiwan, because the members are more active in the participation of the meetings, workshops, and legislation for the disabled to promote their life quality. However, this study may still be helpful for the clinicians and the officials in planning future programs for improving the quality of life of the spinal cord injured patients.

After this survey, the results were reported to the Department of Health, Executive Yuan, Republic of China. Since then, the government and the private enterprises have made several modified interventions to improve the life quality of spinal cord injured patients, such as establishing a computer training program and a practising factory for paraplegic and tetraplegic individuals, in 1995.

\section{Acknowledgements}

The authors thank Ms Hua-Fang Liao, Associate Professor in the School of Physical Therapy, National Taiwan University, for her advice and assistance on statistical analysis. Also, to $\mathrm{Mr} \mathrm{Al}$ Vendouris for his English language editing and consultancy. This investigation was supported by a research grant from Department of Health, Executive Yuan, of Republic of China (No. DOH82-MA056).

\section{References}

1 Geisler WO, Jousse AT, Wynne-Jones M, Breithaupt D. Survival in traumatic spinal cord injury. Paraplegia 1983; 21: 364-373.

2 Weisgerber RA. Quality of life for persons with disabilities. Maryland: An Aspen Pub. 1991: pp1-9.

3 Cushman LA, Hassett J. Spinal cord injury: 10 and 15 years after. Paraplegia 1992; 30: 690-696.

4 DeVivo MJ, Richards JS. Community reintegration and quality of life following spinal cord injury. Paraplegia 1992; 30: $108-$ 112 .

5 Eisenberg MG, Salts CC. Quality of life among aging spinal cord injured persons: Long term rehabilitation outcomes. Paraplegia 1991; 29: $514-520$.

6 Siösteen A et al. The quality of life of three functional spinal cord injury subgroups in a Swedish community. Paraplegia 1990; 28: $476-488$

7 Lawton MP. Environment and other determinants of well-being in older people. The Gerontologist 1983; 23: 349-357.

8 Flanagan JC. Measurement of quality of life: Current state of the art. Arch Phys Med Rehabil 1982; 63: 56-59.

9 Ferrans CE, Powers MJ. Quality of life index: Development and psychometric properties. ANS 1985; 8: $15-24$.

10 Arnold SB. Measurement of quality of life in the frail elderly. Chapt. 3. In: The concept and measurement of quality of life in the frail elderly. Edited by Birren JE, Lubben JE, Rowe JC and Deutchman DE. San Diego: Academic Press, Inc. 1991: pp5073 
11 White MJ et al. Sexual activities, concerns and interests of men with spinal cord injury. Am J Phys Med Rehabil 1992; 71: 225 231 .

12 Chen CF, Lien IN. Spinal cord injuries in Taipei, Taiwan, 1978 1981. Paraplegia 1985; 23: $364-370$.

13 Lan $\mathrm{C}$ et al. Traumatic spinal cord injuries in the rural region of Taiwan: an epidemiological study in Hualien county, 19861990. Paraplegia 1993; 31: 398 - 403.

14 American Spinal Injury Association: Standards for neurologica and functional classification of spinal cord injury, revised 1992. Chicago: ASIA, 1992.

15 Yarkony GM et al. Benefits of Rehabilitation for traumatic spinal cord injury: Multivariate analysis in 711 patients. Arch Nurol 1987; 44: $93-96$
16 Derogatis LR. The psychosocial adjustment to illness scale (PAIS). J Psychosomatic Res 1986; 30: 77-91.

17 Judd FK et al. Psychological adjustment following traumatic spinal cord injury: A study using the Psychosocial Adjustment to Illness Scale. Paraplegia 1991; 29: $173-179$.

18 Trieschmann RB. The philosophy of rehabilitation. Chapt. 2. In Spinal cord injuries: Psychological, social and vocational rehabilitation. Edited by RB Trieschman. 2nd Ed. New York: Demos Pub, 1988: pp25-43. 